\title{
Traffic Reduction in Hybrid Service Discovery Protocol in Mobile Ad-Hoc Grid
}

\author{
Askari Parichehreh \\ Department of Computer Engineering \\ Islamic Azad University of Qazvin \\ Qazvin, Iran \\ apmastermail@gmail.com
}

\author{
Bahman Javadi \\ Department of Computer Engineering \\ Islamic Azad University of Qazvin \\ Qazvin, Iran \\ javadi@aut.ac.ir
}

\author{
Abolfazl Toroghi Haghighat \\ Department of Computer Engineering \\ Islamic Azad University of Qazvin \\ Qazvin, Iran \\ Haghighat@qazviniau.ac.ir
}

\begin{abstract}
Service discovery is an important part of nodes communication and collaboration infrastructure in grid computing and the first stage of grid computing scheduling stages. Thus service discovery is very effective in performance and efficiency of grid. Ad hoc grid is one of the earlier distributed systems that combine capabilities of grid computing and flexibility of ad hoc network. Therefore service discovery protocols have an important role in their efficiency. Up to now, many related work to discovery protocol in mobile ad hoc grid have presented that each of them have had advantages and disadvantages. In this paper we try to control query message by caching them into local query control table to decrease network traffic and enhance efficiency of hybrid service discovery protocol. An experimental result shows that we achieve between 10 to 30 percent improvement in our proposed algorithm.
\end{abstract}

Keywords—mobile ad-hoc grid; service discovery; hybrid protocol;

\section{INTRODUCTION}

Mobile ad-hoc grid is a computational environment that use parallel and distributed technology. Computing nodes in this system establishes on mobile ad-hoc network, so this technology combine capability of traditional grid with flexibility of mobile ad-hoc networks. However, the development of ad-hoc grids entails new challenges, compared to traditional wired grids. Resource discovery, power consumption, QoS security and etc, are problems that have still to be solved. In this paper we study in-depth the problem of resource discovery in mobile ad-hoc grids. Generally, we can classify service discovery mechanism in three main categories:

1- Advertisement based service discovery

2- Query based service discovery

3- Hybrid service discovery

In the advertisement mechanism or proactive model, servers advertise periodically, by broadcasting or multicasting the location and attributes of resources and services. So that client can build a local database with all the services available on the grid environment. Examples of P2P Advertising mechanisms are the Konark passive push protocol [1], and the UPnP discovery service [2].

In query based mechanism or reactive model, client sends a query message to other nodes, by broadcasting or multicasting and ask about services that match same specific attributes. Any servers that have that service respond to the client's query by sending a description of the service, Examples of P2P query-based systems are the Bluetooth
SDP[3], the Konark active pull protocol [1], and the service discovery mechanism proposal for on-demand ad-hoc networks[4],[5].

Hybrid mechanism tries to find a good compromise between proactive and reactive method. The basic idea of hybrid mechanism is to limit the advertisement message within small zone; a zone is defined for any node individually to reduce advertisement overhead and in the other hand enhanced query response time. Query by sending a description of the service, Example of hybrid service discovery can observe in [6].

In this paper we plan to introduce query control mechanisms, which try to avoid that a given node could forward the same query request several times, and to prevent query requests from being forwarded to zones already visited. The remainder of the paper is structured as follows. First of all, we make a survey on hybrid service discovery protocol in section 2. The proposed our query control mechanism is presented in section 3. Simulation environment and results for performance evaluation are presented in section 4. Finally, section 5 concludes this paper.

\section{RELATED WORK}

A considerable amount of work has been spent into studying and designing in ad-hoc grid environments, in this section we want to describe three important service discovery protocols that implemented in real situation.

\section{A. Service location protocol (SLP):}

This protocol was introduced by the IETF SVRLoc Working Group and initiated in 1997. SLP version 2 is the last version of SLP and was standardized in 1999. [12], [13]

SLP is a lightweight, open and scaleable protocol for service discovery in IP networks. It can use several architecture such as centralized directory, decentralized directory and even directory less architectures. SLP also use three agents: user agents (US) that perform service discovery on behalf of clients or applications; service agents (SA), that advertise the service information and position; directory agents (DA) that service information ore registered on it. If DA is present, SPL use centralized architecture and if DA is not present SLP use directory less architecture.

\section{B. Jini:}

Jini was introduced by Sun Microsystems in 1998. The Jini Community was initially established in January 1999 
with the release of the first version of the Jini Technology Starter Kit from Sun Microsystems. [14], [15].

Like to SLP, Jini can provide different architectures. Jini use Look up Service (LUS) as directory agent that maintains a database for all services in the network. When LUS if found, services register with it and client's can queries it with Remote Method Invocation (RMI). Then LUS matches the query against the registered services information and response to the client. A client may receive several responses from several LUS; therefore client may run filtering process in order to make a selection over the received messages.

\section{Konark:}

Konark is a peer to peer service discovery mechanism, each node that provide services runs a SDP manager, SDP managers in device used for two basic function: 1- registering and advertising local services and 2- service discovery on behalf of the clients application. To discover the service Konark use two different models: Active pull and passive push model. In active pull, client's multicast a service discovery message and servers that have a required service response to this message. In passive push model, servers advertise periodically the services that are locally registered. [1]

\section{MODIFICATION ON HYBRID SERVICE DISCOVERY}

\section{A. brief survey on hybrid service discovery}

This protocol is similar to idea that introduced in Zone Routing Protocol [9], [10], [11]. A discovery zone is defined for any node individually, and is composed by the entire neighbor whose distance to the node in question dose note exceeds a certain number of hops, that we named in $\mathrm{R}$ where $\mathrm{R}$ is the zone radius. Service Discovery mechanism in this protocol use hybrid peer to peer mechanism. For service discovery within the zone we used advertisement mechanism and for service discovery between the zones the query mechanism is used. We will describe these two mechanisms in the following.

\section{A.1 Advertising mechanism}

Any node periodically broadcast message to the rest of the node within the discovery zone. These messages that we called advertisement message contain all of the service information that sender node is inclined to share them and service information that other neighbor nodes within the zone inclined share them. This information can be including static and dynamic information related to services. This information showed in table 1.

TABLE I. PARAMETERS IN ADVERTISEMENT MESSAGE

\begin{tabular}{|c|l|}
\hline $\mathbf{N}$ & Service owner node name \\
\hline \hline SN & Services name(Hardware or software service) \\
\hline SD & Available data in node and with service \\
SDD & Descriptions of service and data \\
\hline
\end{tabular}

In advertisement mechanism each node will have a local database that includes information about services that is available in interior and peripheral nodes. This information is collected with messages that forward from neighbors. Service lookup table (SLT) is a table like table 2 that each record of it related to information of one node.

According to Figure 1, when each node interred to grid environment first should became authenticated then it will find its neighbors and send its SLT to them and when a node receive the advertisement message related to other nodes, update self SLT and then send it to other node with in the zone.

TABLE II. SERVICE LOOKUP TABLE

\begin{tabular}{|c|l|l|l|}
\hline $\mathbf{N}$ & SN & \multicolumn{2}{|c|}{ SD } \\
\hline $\mathbf{1}$ & & & \\
\hline $\mathbf{2}$ & & & \\
\hline $\mathbf{3}$ & & & \\
\hline $\mathbf{4}$ & & & \\
\hline $\mathbf{5}$ & & & \\
\hline
\end{tabular}

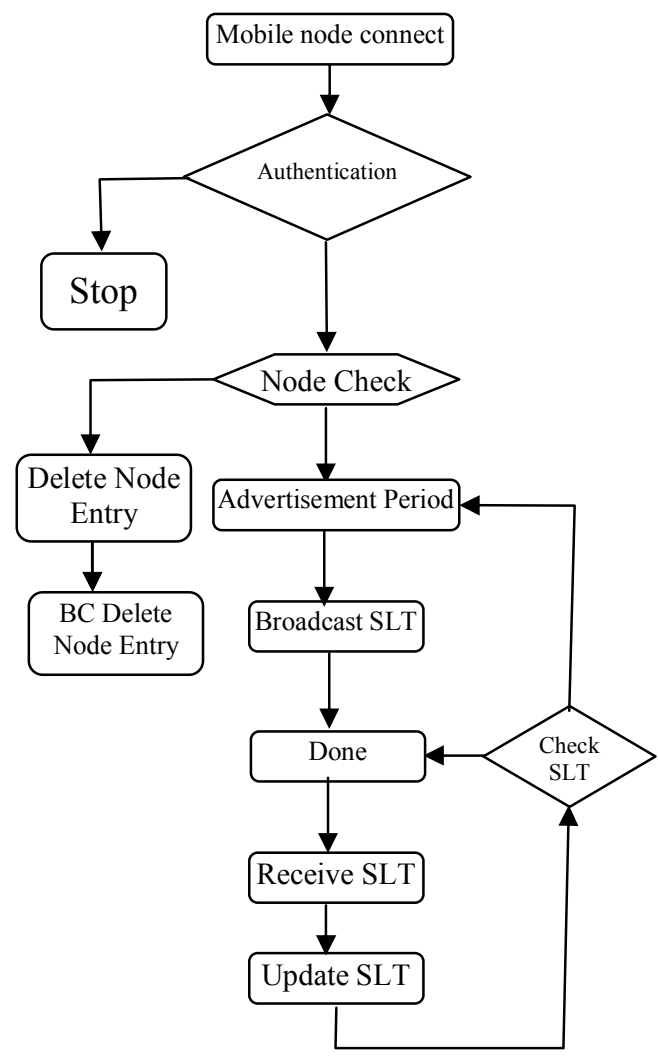

Figure 1. Advertisement mechanism in hybrid protocol 


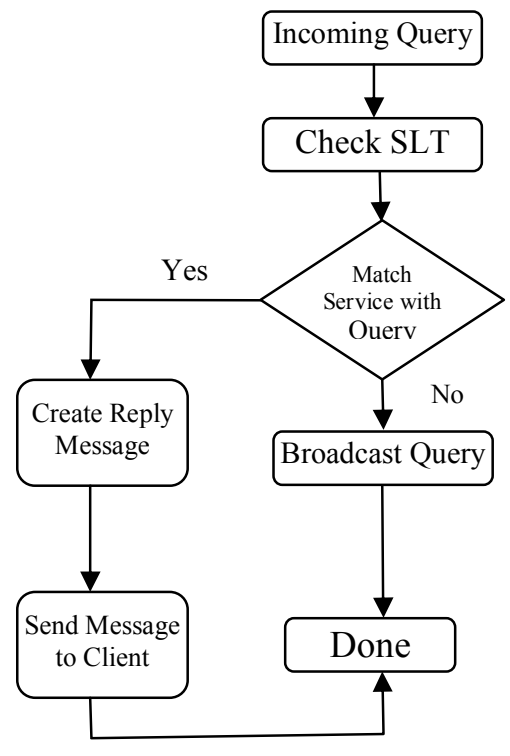

Figure 2. Query mechanism in hybrid protocol

\section{A.2 Query mechanism}

When available services in a zone are not enough for request of applicant node, we must use a query mechanism. In this condition applicant node sends a query to neighbor nodes that take information about available nodes in neighborhood zone. We called this border casting. This operation can repeat with neighbor nodes to achieve information from other far zone and this work can be continued until the requirement of client is provided or service discovery becomes time out. Figure 2 shows status diagram related to query mechanism.

At the end, reply message sent by neighbor nodes to applicant node, for this work in any query message exist a list that include ID of all nodes that received this message and sent to other nodes. By traverse from back to front of the list can find first node that sent the query message. Replay message include a list of services that provide client requirement. Usually a client receives many reply messages; in this case first arrival message will be acceptable.

\section{B. Modified hybrid service discovery}

The idea of hybrid service discovery protocol is that by guiding of query message to neighbor nodes in hop by hop form, can have a better efficiency rather than other protocols (like peer to peer protocol or directory based protocol). But because of overlapping zones maybe a node sends a query many times and receives the same query many times. This leads to more network traffic in the hybrid service discovery rather than other service discovery protocols. To avoid of this event we should develop the query control mechanism and query termination mechanism in this protocol

For better understanding of this matter, we focused on two zones in ad-hoc grid environment. When a node broadcasts a query all nodes in a zone will receive the query and each of them will send it in their zone.

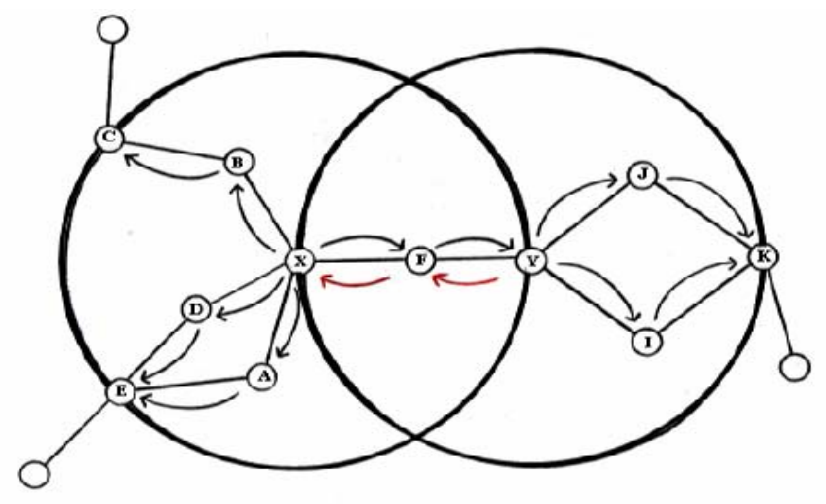

Figure 3. Refused message in two zone

Thus, this process creates more much traffic for answering to this query from other nodes in the grid environment. So we need to design a query control mechanism to control sending messages and forwarding them to appropriate new zone out of present zone.

In continue we present an applicable example of our algorithm. According to Figure 3, we imagine a node X needs a special service in mobile ad-hoc grid and was not found that service in own service lookup table. Thus $\mathrm{X}$ sends a query message to all neighbor nodes (in this figure neighbor nodes are $\mathrm{C}, \mathrm{E}, \mathrm{Y})$. Neighbor nodes evaluate this query message, this work is comparison between available parameters in query and their service lookup table parameters and if the service is available in that node zone like $\mathrm{Y}$ zone, $\mathrm{Y}$ will sent a reply message to $\mathrm{X}$ otherwise if the service is not exist in $\mathrm{Y}$ zone, $\mathrm{Y}$ should border cast the query message to all peripheral nodes in its zone (in this figure the neighbor of $\mathrm{X}$ are $\langle\mathrm{X}, \mathrm{K}>$ ), we can see that $X$ is accounted a part. $X$ receives a query that itself sends it. When $X$ receives and evaluates this query and doesn't have services to reply this query, it send query message again to neighbor nodes, this condition much more critical. Finally, it will be created an extremely loop as $<(\mathrm{X}, \mathrm{F}),(\mathrm{F}, \mathrm{Y}),(\mathrm{Y}, \mathrm{F}),(\mathrm{F}, \mathrm{X})>$ from continuous messages sending which is not suitable for efficient service discovery protocol. We named these messages the confused messages (these messages showed in Figure 6 with red arrow). When this process occurs for all nodes in all zones in grid environment the condition will be more intricate.

Our proposed algorithm in two phases omits the confused messages and for correct omitting messages we must dedicate messages, thus we must be capable to record message information. The first phase of our algorithm must do this work. In this phase, each message that arriving to a node will record in nodes local table. The information that we must record are the message identifier and sender identifier $<$ ID, Message ID>, because, by combination of these two parameters we achieve the unique key for any messages.

In this algorithm, we use another local structure in every node to keep receiving messages information, which we call it QCT and each record of this structure keeps sender ID and message ID. In the second phase, if the message sends for example to $\mathrm{X}$ which has already received and forwarded it, in this case the message will be omit in X. description of second phase will be presented in the following: at the first, for each 
arrival query message; compare its ID and sender ID with the information in QCT and after comparison with any entry of this table if the result shows that it is a confused message, it will be discarded in our modified algorithm.

Modifying of hybrid service discovery protocol just includes applying changes in query mechanisms. Because of did not change in advertisement mechanism of our algorithm, there will not be any problem in management of network and by changing of the topology, service lookup tables will be up to date immediately by advertisement mechanism and for this we do not have any additional work. In continue we show modified statues diagram of query mechanism in Figure 4.

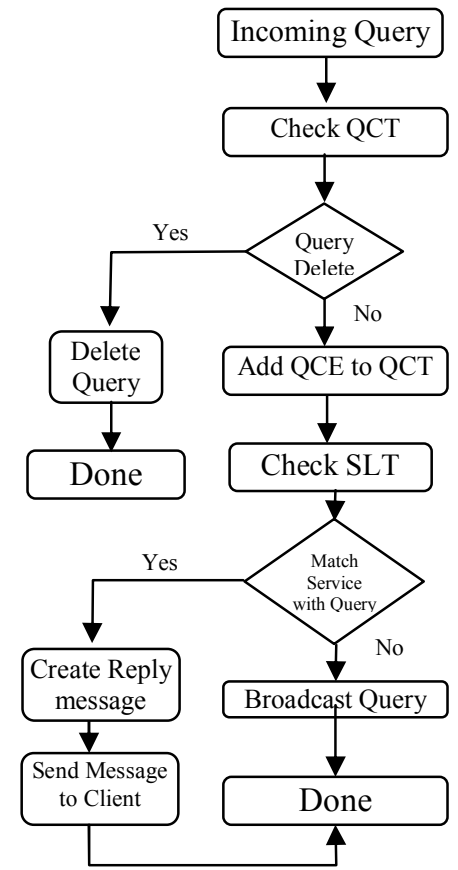

Figure 4. Modified query mechanism

\section{EXPRIMENTAL RESULTS}

We evaluated our modified hybrid service discovery algorithm on Glomosim simulator [16]. Our goal in this section was accounting the number of message that interchange between different ad-hoc grid nodes. We consider zone radius, $\mathrm{R}$ as a variable parameter, as a point, if we take $\mathrm{R}$ equal to zero, the modified protocol will be converged to query based protocol and if R has gone toward extremely, modified protocol will be converged to advertisement protocol. We choose $\mathrm{R}$ as a variable parameter between one and eight. You can see our simulation parameters in table 3 in the following.

\section{TABLE III. PARAMETER'S VALUE IN SIMULATION}

\begin{tabular}{|c|c|c|}
\hline parameter & symbol & value \\
\hline Number of nodes & $\mathrm{N}$ & $100-2500$ \\
\hline Network coverage area & $\mathrm{A}$ & $1000 \mathrm{~m}^{2}$ \\
\hline Node speed & $\mathrm{V}$ & $10 \mathrm{~m} / \mathrm{s}$ \\
\hline Routing zone Radius & $\mathrm{R}$ & $1-8$ \\
\hline
\end{tabular}

Figure 5, Shows the results of my assessments. Number of message is the parameter that shows total messages
(Advertisement message and query message) that interchange between nodes in ad hoc grid.

We compare our algorithm with traditional hybrid service discovery and as shown above, number of sent message in Figure 5-a is less than the same quantitative of Figure 5-b. When we used query message and radius is variable between 0 and 2. For example when we imagine radius is equal to one interchange of query message between nodes in traditional hybrid protocol is 120 messages but in our modified protocol are 100 query messages. This difference between the two numbers is appears because our proposed algorithm just verifies query message and as shown above advertisement message is equivalent in two protocols.

In the following, we used complex scenarios for assessment of modified hybrid protocol. We tried to test our algorithm on mobile ad-hoc network with different nodes (between 100 to 500) and different radius (between 0 to 8). We also compare our algorithm with other algorithm such as P2P-query mechanism, P2P-advertisment mechanism, and traditional hybrid mechanism. Our goal in this section was accounting the number of message that interchange between different nodes.

According to Figure 6, the number of interchanged message in modified status is less than traditional hybrid protocol. Sent messages in modified protocol have 10 to 30 percent improvement than traditional protocol. In smaller zones we achieve near to 30 percent improvement because in these zones query confused and replicated messages are more than bigger zones. Modified protocol has verified on border casting query message between nodes in grid environment and omitted every message that confused and could be replicated. For this reason, sent messages in the modified protocol are less than messages in the traditional protocol.

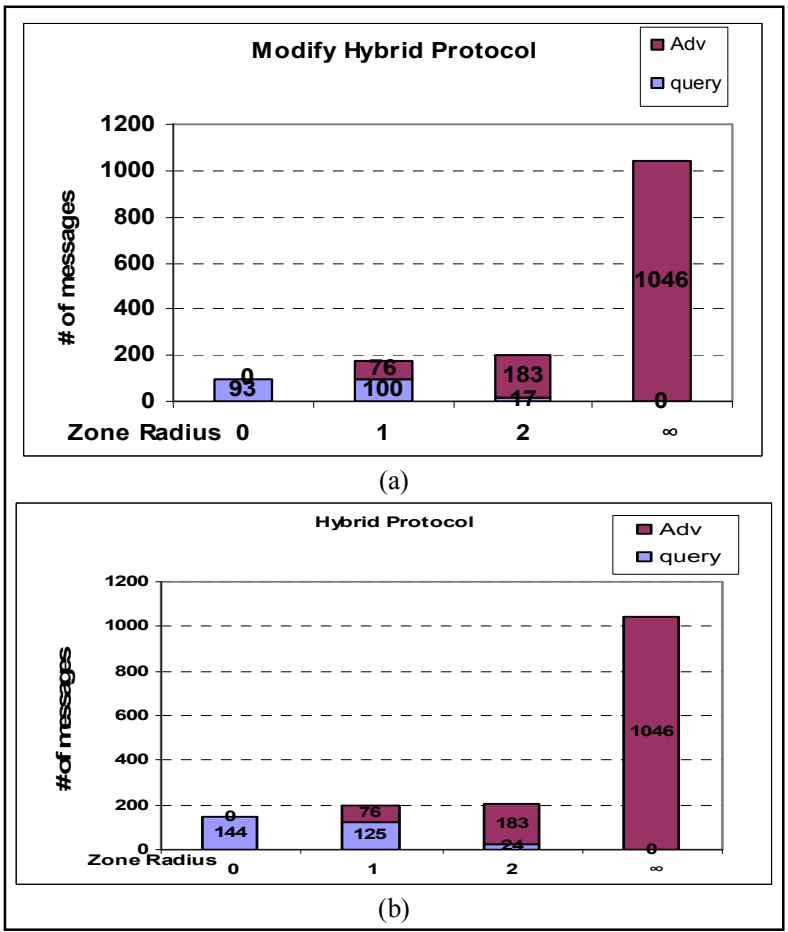

Figure 5. Comparison between number of messages (adv and query) 


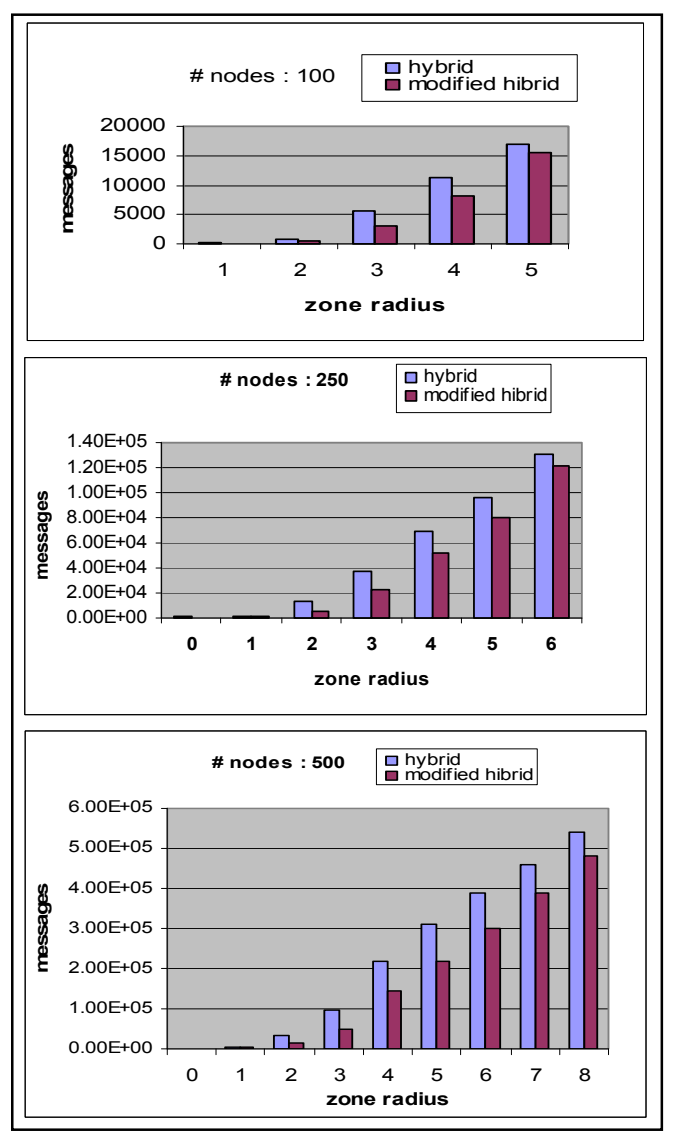

Figure 6. Comparison between traditional hybrid method and modified hybrid method based on number of sent messages

As we mentioned in before, reduction of total message occurred only by reduction of query confused and replicated messages. Because our proposed algorithm is a query control mechanism and does not have affection on advertisement messages. Of course, lower radiuses can be used to reduce number of advertisement messages and avoid from message flooding. But this condition, using lower radius, drives us to query based mechanism that its response time is very high which unfavorable for users.

\section{CONCLUSIONS AND FUTURE WORKS}

Choosing an effective service discovery algorithm is a big challenge in the mobile ad-hoc grid. Generally we can divide the service discovery protocols to three classes: 1- peer to peer mechanism that had a good adaption to mobile ad-hoc environment and had a high flexibility against topology dynamicity, but didn't have a good scalability. 2- Directory based mechanism that had a good scalability, but didn't have a good flexibility against topology dynamicity. 3- Hybrid mechanism that was combination of two upper methods but had some problems.

In this paper we presented a query control mechanism to improve efficiency of hybrid protocol; we can get a lower number of interchange messages by avoiding of sending confused and replicated queries. According to results of simulation, we reduce the bandwidth consumption and number of messages that has been interchanged between nodes in ad-hoc grid environment.

As a future work, we will try to design a more reliable service discovery protocol based on node mobility and life time of device power to improve hit ratio of successful service delivery.

\section{REFERENCES}

[1] S. Helal, N. Desai, V. Verma, C. Lee, "Konark - A Service Discovery and Delivery Protocol for Ad-hoc Networks", in: Proc.of the Third IEEE Conference on Wireless Communication Networks (WCNC), New Orleans, March 2003.

[2] B.A. Miller, T. Nixon, C. Tai, M.D. Wood," Home networking with universal plug and play", IEEE Communications Magazine 39 (12) (2001) 104-109.

[3] E.A. Gryazin, "Bluetooth. Service discovery protocol", Bluetooth Specification Version 1.1, Part E, Feb. 2001.

[4] R. Koodli, C. Perkins, "Service discovery in on-demand ad hoc networks", IETF Internet Draft (draft-koodli-manet servicediscovery00.txt), October 2002

[5] C. Frank, H. Karl, "Consistency challenges of service discovery in mobile ad hoc networks", in: Proc. of the 7th ACM Int. symposium on modelling, analysis and simulation of wireless and mobile systems (MSWiM), 2004, pp. 105-114.

[6] R. Moreno, "A hybrid mechanism for resource/service discovery in adhoc grids", Dept. de Arquitectura de Computadores y Autom'atica, Universidad Complutense de Madrid, 28040 - Madrid, Spain. 2008

[7] T. Phan, L. Huang, C. Dulan. "Challenge: Integrating Mobile Wireless Devices into the Computational Grid".2003, pp. 72-81.

[8] Z. Li, L. Sun, "Challenges Of Mobile Ad-Hoc Grids and Their Applications in E-Healthcare", University of Plymouth, UK 2005.

[9] Z. J. Hass, "A new routing protocol for the reconfigurable wireless networks," Proceedings, IEEE 6th International Conference on Universal Personal Communications, 1997, pp. 562-566.

[10] Z. J. Hass and M. R. Pearlman, "The performance of a new routing protocol for the reconfigurable wireless networks," Proceedings, IEEE International Conference on Communications (ICC), 1998, pp. 156160.

[11] M. R. Pearlman and Z. J. Hass, "Determining the optimal configuration for the zone routing protocol," IEEE Journal on Selected Areas in Communications, vol. 17, no. 8, pp. 1395-1414, Aug. 1999.

[12] E. Guttman, C. Perkins, J. Veizades, and M. Day, "RFC 2608: Service location protocol, version 2," 1999, status: PROPOSED STANDARD.

[13] SRVLOC, "Service Location Protocol (svrloc) Working Group," 1997.

[14] Sun Microsystems, "Device Architecture Specification," Sun Microsystem, Inc, Tech. Rep., June 2003.

[15] Sun MicroSystems, "The Davis Project," 2003. [Online]. Available: http://davis.jini.org/

[16] Glomosim simulator homepage, http:// pl.cs.ucla.edu/projects /glomosim 